\title{
Flow Reduced in Coronary Microcirculation
}

National Cancer Institute

\section{Source}

National Cancer Institute. Flow Reduced in Coronary Microcirculation. NCI Thesaurus.

Code C119219.

A finding of decreased blood flow in the microcirculation of the heart. 